\title{
Structure and evolution of online social relationships: Heterogeneity in unrestricted discussions
}

\author{
K.-I. Goh, ${ }^{1, *}$ Y.-H. Eom, ${ }^{2}$ H. Jeong, ${ }^{2}$ B. Kahng, ${ }^{1,3}$ and D. Kim ${ }^{1}$ \\ ${ }^{1}$ School of Physics and Center for Theoretical Physics, Seoul National University, Seoul 151-747, Korea \\ ${ }^{2}$ Department of Physics, Korea Advanced Institute of Science and Technology, Daejon 305-701, Korea \\ ${ }^{3}$ Center for Nonlinear Studies, Los Alamos National Laboratory, Los Alamos, New Mexico 87545, USA
}

(Received 21 February 2006; published 20 June 2006)

\begin{abstract}
With the advancement in the information age, people are using electronic media more frequently for communications, and social relationships are also increasingly resorting to online channels. While extensive studies on traditional social networks have been carried out, little has been done on online social networks. Here we analyze the structure and evolution of online social relationships by examining the temporal records of a bulletin board system (BBS) in a university. The BBS dataset comprises of 1908 boards, in which a total of 7446 students participate. An edge is assigned to each dialogue between two students, and it is defined as the appearance of the name of a student in the from- and to-field in each message. This yields a weighted network between the communicating students with an unambiguous group association of individuals. In contrast to a typical community network, where intracommunities (intercommunities) are strongly (weakly) tied, the BBS network contains hub members who participate in many boards simultaneously but are strongly tied, that is, they have a large degree and betweenness centrality and provide communication channels between communities. On the other hand, intracommunities are rather homogeneously and weakly connected. Such a structure, which has never been empirically characterized in the past, might provide a new perspective on the social opinion formation in this digital era.
\end{abstract}

DOI: 10.1103/PhysRevE.73.066123

PACS number(s): 89.75.Fb, 89.65.-s, 87.23.Ge

\section{INTRODUCTION}

With the advancement in the information age, people are using electronic media for communication more frequently, and social relationships between people are also increasingly resorting to online communications. For example, the advent of online bulletin board systems (BBS) made it possible to develop a new type of an online social relationship and social consensus. Very similar to the Usenet service, which was fairly popular during the earlier days of the internet, BBS is based on the communication between people sharing common interests; the topic of interest is usually identified by the board itself. People with common interests post messages on a certain board and a response is conveyed by posting another message, thereby forming a thread. Thus, a thread in the BBS roughly represents a dialogue between people, and such a dialogue constitutes the basic relationship among the people participating in it. In the BBS, dialogues or discussions usually proceed with little restriction on message writing and discrimination based on personal information, thereby forming the so-called "warm" discussions as described in psychosociology [1]. Therefore, the pattern of such online social relationships may be different from that of traditional social relationships based on face-to-face contact or online communication involving an exchange of personal information, such as e-mail transactions [2-6] and instant messaging [7]. Thus, it would be interesting to study the structure of online social relationship networks constructed

\footnotetext{
*Present address: Center for Cancer Systems Biology, DanaFarber Cancer Institute, Boston, MA 02115, USA and Department of Physics, University of Notre Dame, Notre Dame, IN 46556, USA.
}

by people in warm discussions; this would be useful in resolving diverse sociological and political issues and understanding the manner in which social opinion is formed in the digital era [8-12]. Extensive studies on traditional social networks have been carried out [13-15]; however, few studies exist on online social networks. Here, we investigate the structure of online social networks by studying BBS networks, which are familiar to university students.

From the graph theoretical perspective, the BBS network offers distinct features such as weighted and modular network structure. Since the number of times a given pair of people exchange dialogues can be counted explicitly, a weighted network is naturally obtained [16]. Moreover, since people are sharing a board corresponding to their common interests, BBS provides an unambiguous way of defining modules or communities [17]. This is unlike other examples of accessible protocols, including the sibling and/or peer relationship in the online community [18] and trackback in the blog system [19]. In fact, the BBS network constructed by us differs in crucial aspects from other affiliation networks such as the collaboration network [20] and student course registration network [21]. In these examples, the relationship between people is not explicitly defined but is indicated indirectly by their affiliation. Such an indirect definition generates several cliques, completely connected subgroups, which may result in an artifact particularly in the case of large-sized affiliations. Thus, to obtain a network of people with explicit pairwise interaction strength together with a distinct community definition is crucial for an appropriate description of the social system. The BBS network provides such ingredients.

The BBS network has interesting structural features and implications. It contains hub members who participate in dialogues across a large number of boards, thereby connecting one group of people at one board to another group at a dif- 

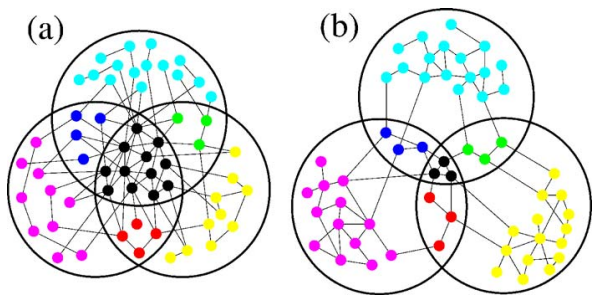

FIG. 1. (Color online) Schematic network snapshots of the BBS network (a) and traditional social network (b).

ferent board. Further, their degrees, which are the numbers of people that they have exchanged dialogues with, are large, thereby influencing other people throughout different communities. As a result, the hub members act as weak ties in connecting different communities; however, their links are strong during an actual activity. On the other hand, intraboard connections are rather homogeneous in degree. Such a network feature is in contrast to traditional social networks maintained by the ties bridging disparate communities, which tend to be weak, as proposed by Granovetter [14]. The difference is schematically depicted in Fig. 1. We also attempt to understand the BBS network from the perspective of a simple network model. In the model, we take into account the empirical fact that the BBS network contains groups of which size are inhomogeneous. In addition, the link density of each group is not uniform, however decreases with increasing group size, which has been usually neglected when a model is constructed. The model constructed in this way is successful in reproducing the BBS network.

\section{BBS NETWORK}

We mainly examined the BBS system at the Korea Advanced Institute of Science and Technology; it is named as loco.kaist.ac.kr. The characteristics of the network structure obtained from this BBS system also appear in another system, bar.kaist.ac.kr. The data comprise records of all the threads posted from 9 March 2000 to 2 November 2004, thus corresponding to a duration of around three and a half years. As of November 2004, the system comprised 1908 boards with a total of 7446 participating students. In order to ensure privacy, we are only allowed to access the information on "from," "to," the date of posting, and the name of the board it was posted on, for each message. Most of large boards in this BBS are found to be of personal blog-type, but it also include community or topical boards, such as a university club or alumni union. Based on this information, we constructed the network between students such that for each message, an edge was assigned between two students appearing as from and to. Alternatively, an arc (a directed edge) can be assigned for each message; however, we found that the communications are largely reciprocal: Approximately a half of the postings are accompanied by another one with its from and to fields reversed, for example, a "Re:" message. Subsequently, we shall consider the network as undirected for simplicity.

Our network construction naturally yields a weighted network in which the weight $w_{i j}$ of the edge between two stu-
TABLE I. Statistics of the BBS network as of November 2004. The numbers in parentheses are the statistics for non-self-dialogs.

\begin{tabular}{ll}
\hline \hline Number of students $N$ & $7446(7421)$ \\
Number of links $L$ & $103498(103473)$ \\
Number of dialogs $W$ & $1299397(1267292)$ \\
Number of boards $G$ & $1908(1872)$ \\
Size of the largest cluster $N_{1}$ & 7350 \\
Average size of the boards $\bar{S}$ & $32.0(32.6)$ \\
Average board memberships of & 8.2 \\
a student $\bar{B}$ & \\
Average path length $D$ & 3.3 \\
Mean degree $\langle k\rangle$ & $27.8(27.9)$ \\
\hline \hline
\end{tabular}

dents $i$ and $j$ is determined by the number of messages they exchanged during the period. The detailed statistics of the BBS are listed in Table I.

\section{STRUCTURE OF THE BBS NETWORK}

\section{A. Student network}

The global snapshot of the student network in Fig. 1 reveals the inhomogeneity among the students. The degree $k_{i}$ of a student $i$, which is the number of students he and/or she has exchanged dialogues with, is distributed according to a power law with an exponent of around -1 followed by an exponential cutoff, as shown in Fig. 2(a). This feature is similar to that of the scientific collaboration network [20]. The strength $s_{i}$ of a student $i$ is the sum of the weight of each edge attached to $i$. Therefore, $s_{i}=\sum_{j}^{N} a_{i j} w_{i j}$, where $a_{i j}$ is the component of the adjacent matrix; its value is 1 if an edge is connected between vertices $i$ and $j$ and 0 otherwise. $w_{i j}$ is the weight of the edge between $i$ and $j$. The strength and degree of a student exhibit a scaling behavior $s(k) \sim k^{\beta}$ with $\beta$ $\approx 1.4$; however, the fluctuation is quite strong,particularly for a small $k$ [Fig. 2(b)]. The strength distribution exhibits a behavior that is similar to that of the degree distribution; however, the value of the cutoff is larger [Fig. 2(a)]. The nonlinear relationship between $s$ and $k$ implies that the hub members tend to post messages considerably more frequently than the other people, as is evident in Table II.

Other standard measures of network topology are also obtained. The local clustering coefficient $c_{i}$ is the local density of transitive relationships, defined as the number of triangles formed by its neighbors, cornered by itself, $i$, divided by the maximum possible number of these, $k_{i}\left(k_{i}-1\right) / 2$. The average of $c_{i}$ over vertices with a given degree $k$ is referred to as the clustering function $C(k)$. For the student network, $C(k)$ decays as $\sim k^{-0.5}$ for large $k$, and its weighted version defined in Ref. [16] ${ }^{1}$ behaves as

\footnotetext{
${ }^{1}$ In Ref. [16], the local weighted clustering coefficient was defined as $c_{i}^{(w)}=\sum_{j, h}\left(w_{i j}+w_{i h}\right) a_{i j} a_{i h} a_{j h} /\left[2 s_{i}\left(k_{i}-1\right)\right] . C^{(w)}(k)$ is the average of $c_{i}^{(w)}$ over vertices with degree $k$. The weighted average nearestneighbors degree of vertex $i$ was defined as $k_{\mathrm{nn}, i}^{(w)}=\sum_{j=1}^{N} a_{i j} w_{i j} k_{j} / s_{i}$. $k_{\mathrm{nn}}^{(w)}(k)$ is the average of $k_{\mathrm{nn}, i}^{(w)}$ over the vertices with degree $k$.
} 

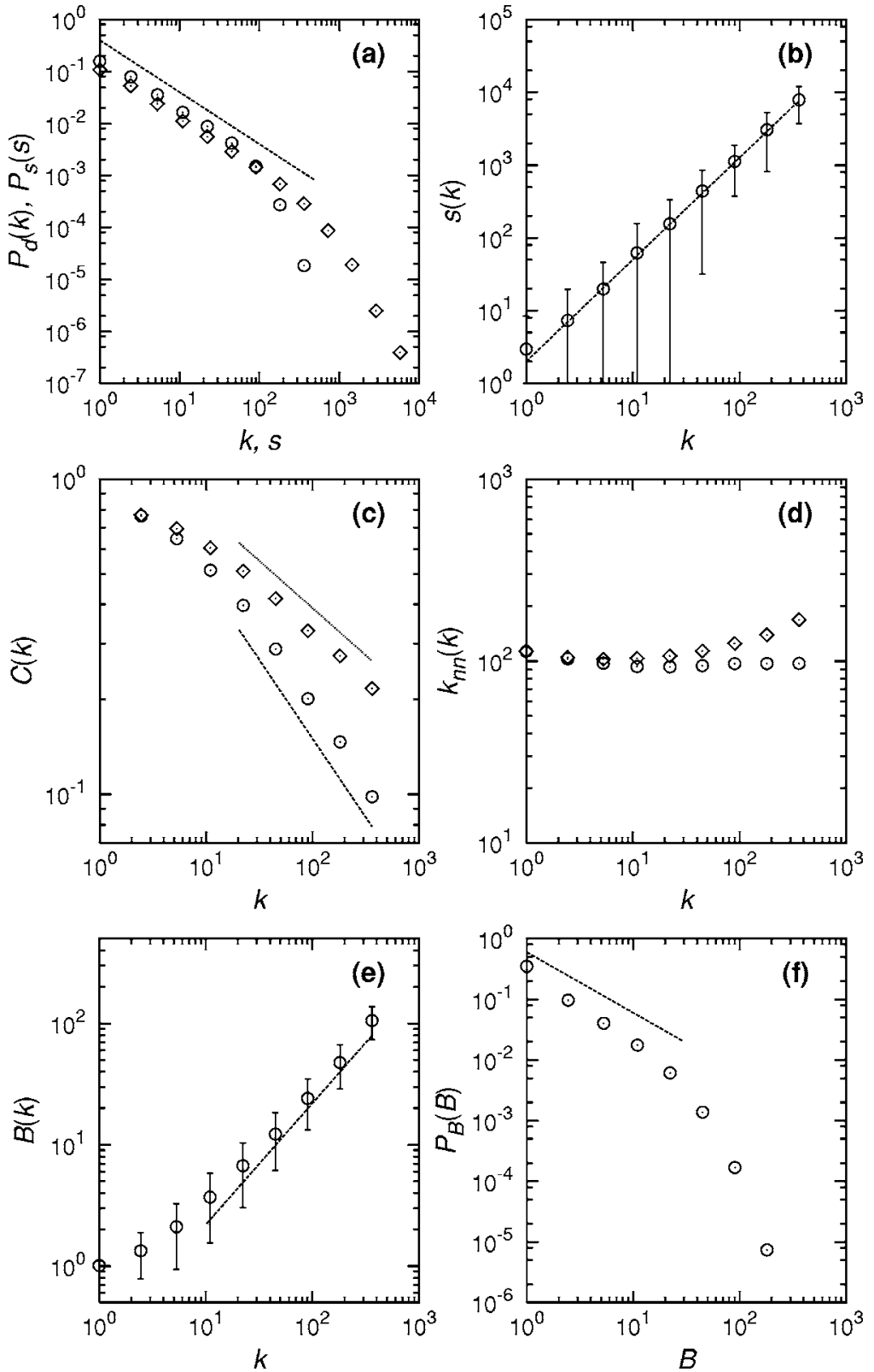

FIG. 2. Structure of the BBS network. (a) The degree distribution $P_{d}(k)(\circ)$ and the strength distribution $P_{s}(s)(\diamond)$ of the entire network. The straight line is a guideline with a slope of -1 . (b) The degree-strength scaling relation $s(k)$. The straight line is a guideline with a slope of 1.4. (c) The clustering function $C(k)(\circ)$ and its weighted version $(\diamond)$. The straight lines are guidelines with slopes of -0.5 (lower) and -0.3 (upper), respectively. (d) The average nearest-neighbor degree function $k_{\mathrm{nn}}(k)$ and its weighted version $(\diamond)$. (e) The correlation between the degree and the membership number $B$. The dotted line is a guideline with a slope of 1 . (f) The membership number distribution of the vertices $P_{B}(B)$, where $B$ is the number of boards that a student participates in. The straight line is a guideline with a slope of -1 .
$C^{(w)}(k) \sim k^{-0.3}$, as shown in Fig. 2(c). The clustering coefficient $C$, which is the average of $c_{i}$ over all vertices with $k>1$, is $\approx 0.48$. This is one order of magnitude greater than $C_{\text {random }} \approx 0.04$ of its typical randomized counterpart with an identical degree sequence [22]. The average nearest-neighbor degree function $k_{\mathrm{nn}}(k)$, which is defined by the average degree of the neighbors of vertices of degree $k$, is almost flat for the student network; nevertheless, its weighted version defined in [16] shows a slightly upward curvature for large $k$ [Fig. 2(d)]. The assortativity coefficient [23] for the binary network and the Spearman rank correlation of the degrees are measured to be close to zero, as $r \approx 0.011$ and $r_{\text {Spearman }} \approx 0.024$, respectively. This almost neutral mixing, which is in contrast to the common belief that social networks are assortative, has also been observed in another online social network [18].

The number of boards that a student participates in is likely to be larger for students with a larger degree, as shown in Fig. 2(e). Its distribution follows a skewed functional form in Fig. 2(f). These results imply an important fact that a group of people with a large degree tend to participate in diverse dialogues on different boards and will play a dominant role in drawing social consensus on diverse issues. Moreover, they work as mediators between different groups in an online social community.

The betweenness centrality (BC) or load [24-26], which is defined as the effective number of paths or packets passing through a given vertex when every pair of vertices gives and receives information, is also measured. The $\mathrm{BC}$ distribution follows a power law with an exponent $\approx 2.2$, as shown in Fig. 3(a) and the BC of a given vertex $\ell$ is strongly correlated to its degree $k$ as $\ell \sim k^{1.6}$ as shown in Fig. 3(b). This implies that the hub members have a large $\mathrm{BC}$ and have a strong influence on the remaining people.

In other words, the student network is extremely heterogeneous, highly clustered, and yet, almost neutrally mixed, 
TABLE II. The fraction of the dialogs contributed by hub members with a degree larger than 80 in the first ten longest threads. The degree value of 80 is chosen approximately in Fig. 2(a); beyond this degree, the power law for the degree distribution fails.

\begin{tabular}{lccc}
\hline \hline Rank & $\begin{array}{c}\text { Thread } \\
\text { length }\end{array}$ & $\begin{array}{c}\text { Number of dialogs } \\
\text { contributed by } \\
\text { hub members }\end{array}$ & $\begin{array}{c}\text { Fraction } \\
(\%)\end{array}$ \\
\hline 1 & 229 & 181 & 79 \\
2 & 121 & 70 & 58 \\
3 & 92 & 92 & 100 \\
4 & 74 & 45 & 61 \\
5 & 67 & 16 & 24 \\
6 & 66 & 45 & 68 \\
7 & 65 & 27 & 41 \\
8 & 64 & 34 & 53 \\
9 & 54 & 54 & 100 \\
10 & 50 & 50 & 100 \\
\hline \hline
\end{tabular}

thereby exhibiting a strong nonlinear relationship between the strength and degree.

\section{B. Board network}

The procedure for constructing the board network is similar to the usual projection method of the bipartite affiliation network. We create a link between two boards if they share at least one common member. In other words, each student participating in more than one board contributes a complete subgraph - a clique - to the board network. Thus, the board network is the superposition of cliques, each of which originates from the crossboard activities of a student. Such crossboard activities will provide channels for information transmission across the boards. In order to assign meaningful weights to these channels, all the links in each clique are assigned a weight that is equal to the inverse of the number of vertices in that clique. In other words, the communication channels created by the students posting on fewer boards are stronger. Therefore, the weight of an edge between two boards increases with the number of comembers; however, the contributions of "ubiquitous persons" would only be moderate. The strength of a board is the sum of the weights of its edges. Such a strength distribution along with the degree distribution, which does not account for the weight, is shown in Fig. 4(a). The relation between the strength and degree is shown in Fig. 4(b).

The board network is quite highly clustered with a clustering coefficient of $\approx 0.61$, and the clustering function decreases with $k$ [Fig. 4(c)]. However, it is worth noting that such a high clustering may result from the generation of cliques by the projection procedure. Moreover, even the randomized board network has a clustering coefficient as high as $\approx 0.48$. The average nearest-neighbor degree initially increases with $k$ but decreases for larger $k$. However, its weighted version increases monotonically with $k$, as shown in Fig. 4(d).

\section{STUDENT NETWORK WITHIN A BOARD}

Upon examining the networks within a board, we were presented with a different scenario. As shown in Fig. 5(a), the degree distributions of the student networks within the boards are rather homogeneous. They exhibit a peak followed by an exponential tail, which overall fits well into the gamma distribution. Here, the degree $k$ must be specified in further detail. Consider a case where two students $A$ and $B$ on a given board do not communicate directly with each other. However, this communication between $A$ and $B$ can occur on a different board. In this case, the two students are regarded to be connected for the definition of degree in Fig. 5(a). When such a pair is regarded to be disconnected, the degree $k_{0}$ is redefined and its distribution exhibits fat tails, as shown in Fig. 5(b); this was also observed in another BBS system [27].

The size of the board, which denotes the number of students posting messages on it, has a broad distribution [Fig. 5(c)] - a power law followed by a rapidly decaying tail. The edge density $\Lambda$ inside a given board scales with its size $M$ as $\Lambda(M) \sim M^{-0.65}$, as shown in Fig. 5(d). Such a behavior cannot be observed in the random sampling of populations of different sizes, thereby indicating that the communications between students are indeed strongly constrained within each board rather than across them. Further, the power-law scaling behavior suggests that the BBS network is organized in a self-similar manner. From this result, it is evident that the usual projection method involving the creation of cliques by
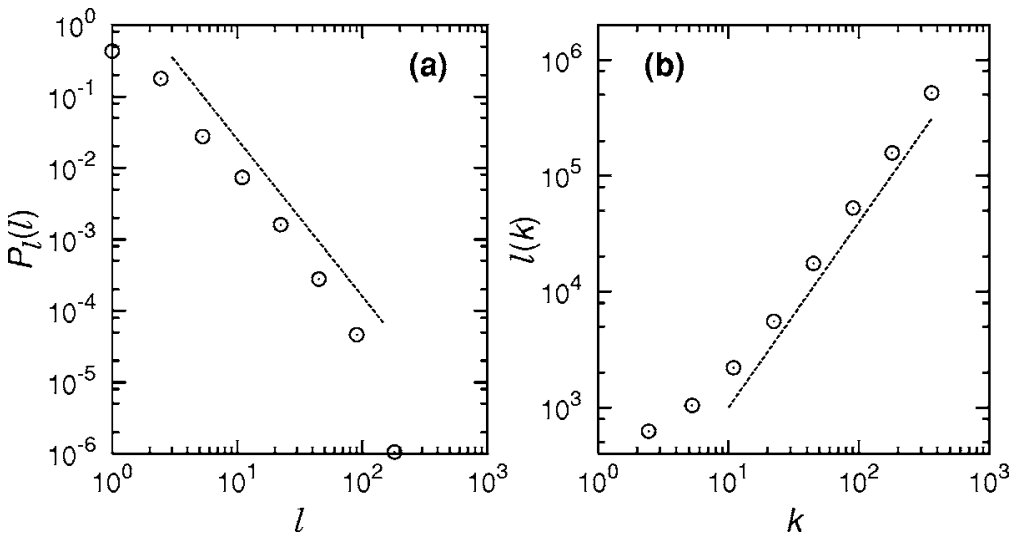

FIG. 3. (a) The betweenness centrality (BC) distribution of the BBS network. The dotted line is a guideline with a slope of -2.2 . (b) The relation between $\mathrm{BC}(\ell)$ and degree $(k)$ of the BBS network. The dotted line is a guideline with a slope of 1.6. 

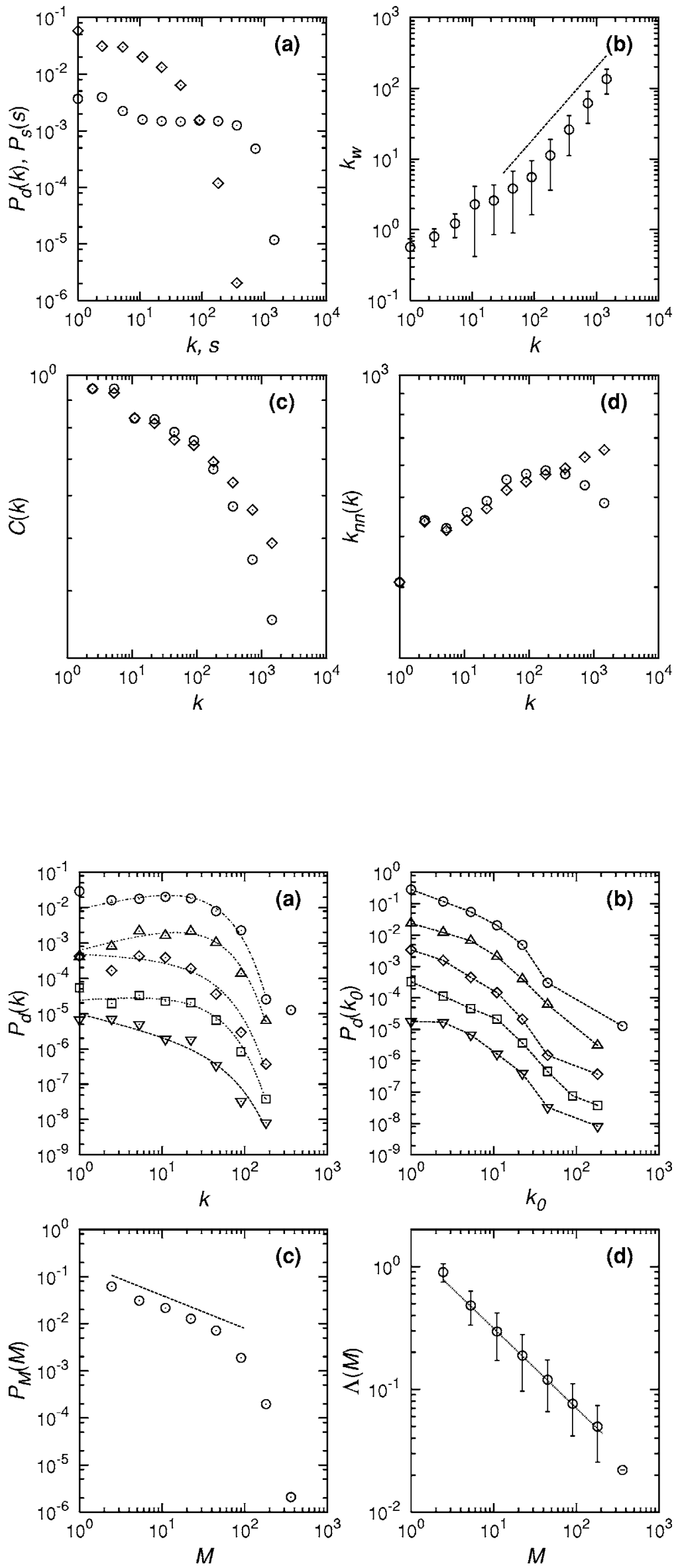

FIG. 4. Structure of the board network. (a) The degree distribution $P_{d}(k)(\circ)$ and strength distribution $P_{s}(s)(\diamond)$ of the board network. (b) The degree-strength relation in the board network. The straight line is a guideline with a slope of 1 . (c) The clustering function $C(k)(\circ)$ and its weighted version $(\diamond)$. (d) The average nearestneighbor degree function $k_{n n}(k)(\circ)$ and its weighted version $(\diamond)$.
FIG. 5. Properties of the board subnetwork. (a) The degree distributions of subnetworks within the five largest boards. Symbols used are $(\circ),(\triangle),(\diamond),(\square)$, and $(\nabla)$ in the decreasing order of board size. The fitted curves with the gamma distribution $k^{a-1} e^{-k / b} /\left[\Gamma(a) b^{a}\right]$ are shown. (b) The degree distributions of subnetworks within the five largest boards with degrees redefined as discussed in the text. (c) The size distribution of the boards $P_{M}(M)$. The straight line is a guideline with a slope of -0.7 . (d) The link density $\Lambda(M)$ within a board as a function of its size $M$. The straight line is a guideline with a slope of -0.65 . 

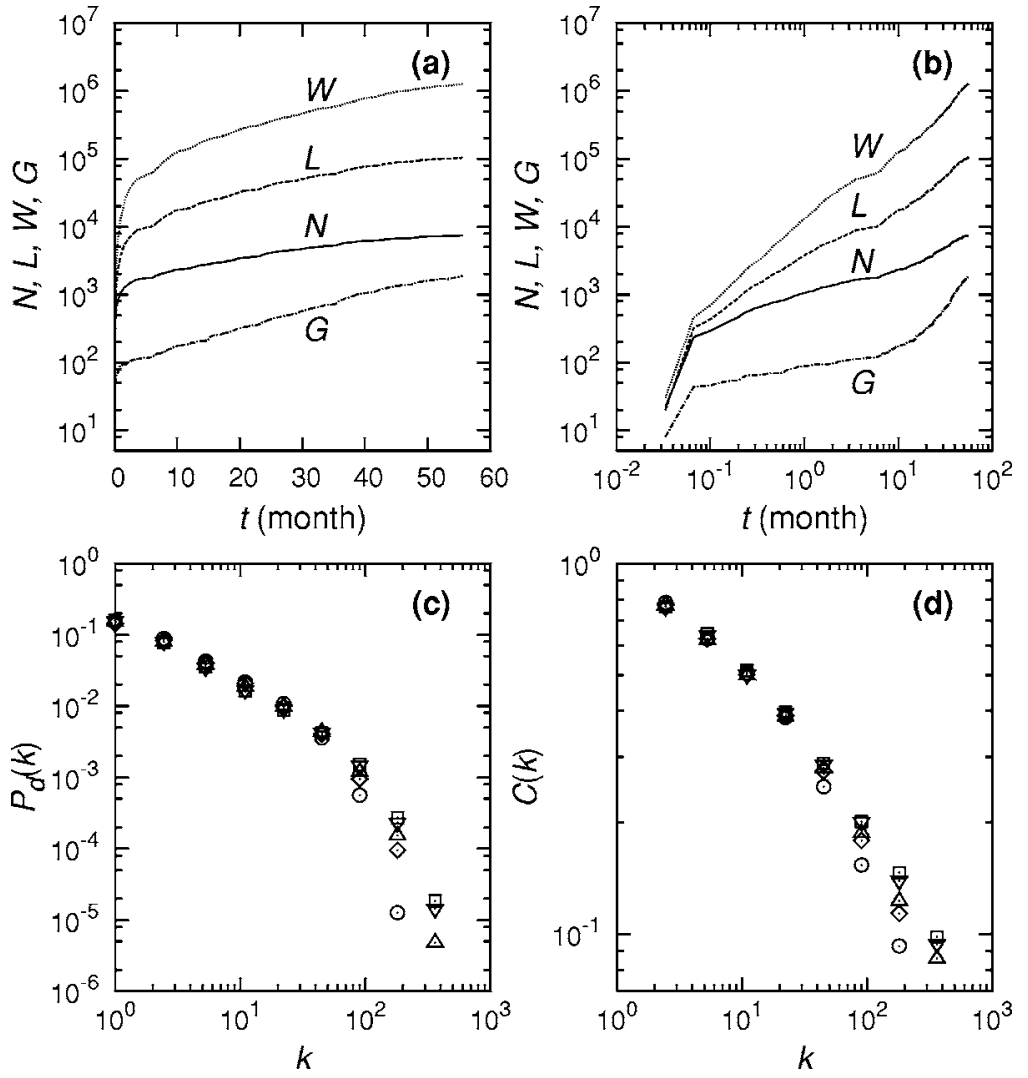

FIG. 6. Evolution of the BBS network. (a) The temporal evolution of the number of students $N$ (solid), number of links $L$ (dashed), total number of dialogs $W$ (dotted), and the number of boards $G$ (dot-dashed). (b) The same plot as (a) in the double logarithmic scale. (c) The evolution of the degree distribution $P_{d}(k)$ of the student network. The degree distribution for each year is shown. The symbols $(\circ),(\diamond),(\triangle)$, and $(\nabla)$ correspond to each year from 2001 to 2004, respectively, and $(\square)$ represents the final configuration. (d) The clustering function $C(k)$ for each year. The same symbols as those in (c) are used. bipartite affiliation graphs cannot provide an appropriate description of the BBS system. Moreover, such a sizedependent scaling of edge density within groups has not been realized thus far in a simple model of a clustered network [28].

\section{EVOLUTION OF THE BBS NETWORK}

The daily record of the BBS network also allows us to examine the temporal evolution of the network. The number of vertices (students) $N$ grows exponentially after the transient period; however, the continuously moderated growth rate appears to attain a steady state [Fig. 6(a)]. Similar behavior is observed in the case of the number of links $L$ and the number of dialogues $W$. The number of boards $G$ grows at a rather steady rate over the period. Preliminary analysis of the temporal evolution at the individual level shows a tendency that older nodes may acquire more degrees on average but with a distributed growth rate. A detailed analysis will be presented elsewhere [29].

Despite its continuous evolution, the structural properties of the network seem to be in a stationary state. In other words, the overall network characteristics such as the degree distribution and clustering function achieve their forms in the initial period (after $\sim 1$ year), and do not change considerably with time, as shown in Figs. 6(c) and 6(d). The crossover time scale of approximately 1 year can also be observed in terms of the evolution of the number of vertices $N$ : Their growth patterns change qualitatively after $\sim 10$ months, as seen in Figs. 6(a) and 6(b). We also found that the hubs became rather stable, in that most of hub nodes identified from the whole history remain to be hubs identified from the last year's activity only.

\section{SIMPLE MODEL}

Having identified the main statistical characteristics of the BBS network, we attempt to understand them from the perspective of a simple network model. First, we consider a simple extension of the model of a clustered network introduced by Newman [28]. The original model of Newman is specified with two fundamental probability distributions, $r_{m}$ and $s_{M} . r_{m}$ represents the probability that an individual belongs to $m$ groups $\left(P_{B}(B)\right.$ in our notation; [see Fig. 5(d)]) and $s_{M}$, the probability that the group size is $M\left[P_{M}(M)\right.$ in our notation]. By assuming that the link density within the groups is given by a constant parameter $p$, it is possible to obtain several of formulas for the network structure using the generating function method. For example, the degree distribution of the network can be written as follows:

$$
P_{d}(k)=\left.\frac{1}{k !} \frac{d^{k}}{d z^{k}} f_{0}\left[g_{1}(p z+q)\right]\right|_{z=0},
$$

where $f_{0}(z)$ and $g_{1}(z)$ are appropriate generating functions defined as $f_{0}(z)=\sum_{m=0}^{\infty} r_{m} z^{m}$ and $g_{1}(z)=\langle M\rangle^{-1} \sum_{M=0}^{\infty} M s_{M} z^{M-1}$, and $q=1-p$.

However an obvious shortcoming of the model is that in real data, the link densities are not uniform across the boards and they strongly depend on the board size, as shown in Fig. $5(d)$. In fact, by simply applying this model with the average link density $p \approx 0.3$ along with $r_{m}$ and $s_{M}$, directly measured 


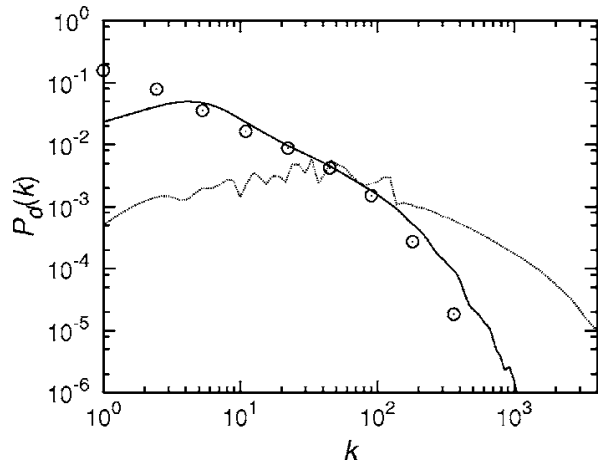

FIG. 7. Comparison of the degree distributions of a simple model of the BBS network of Newman (dotted) and its modification (solid), with that of the real network (circle).

from the data, the degree distribution of the BBS network cannot be reproduced. Therefore, we modify the model by allowing $p$ to vary across the group, based on the empirical formula $\Lambda(M) \sim M^{-0.65}$. Such a modification complicates the mathematical formulas and they must be solved numerically. The resulting degree distribution of the modified model along with that of the real data is shown in Fig. 7. Although it is imperfect, the agreement improved significantly. Thus, it is crucial to incorporate the nonuniform link density into the realistic modeling of the BBS network.

The manner in which the group size distribution, group membership distribution, and group density scaling, which are the input parameters of the model, achieve their present forms, as shown in Figs. 5(c) and 5(d), is a topic for future study.

\section{CONCLUSIONS AND DISCUSSION}

The BBS network contains hub members who participate in dialogues across a large number of boards, thereby connecting one group of people at one board to another group at a different board. Further, their degrees are large, thereby influencing other people throughout different communities. As a result, the hub members act as weak ties in connecting different communities; however, their links are strong during on actual activity. Such a feature has recently been observed also in the bug-reporting communication patterns in online software development community [30]. On the other hand, intraboard connections are rather homogeneous in degree. Such a network feature is in contrast to traditional social networks maintained by the ties bridging disparate communities, which tend to be weak. In the BBS network, the strength $s$, i.e., the total number of dialogues each individual participates in has a nonlinear relationship with the degree $k$ as $s \sim k^{1.4}$. This implies that the hub members tend to post messages at considerably more frequently than the other people with small degrees. The neutrality in the assortative mixing is another feature of the BBS network compared with the assortativity in traditional social networks. Such a behavior may originate due to the absence of personal information on the partner during online social communication. Thus, hub members are democratic in their connections to the remaining people, and they are indeed "ubiquitous persons." Since the hub members play a dominant role in providing communication channels across different boards, it might be more efficient to use a BBS-like online media for persuading people and drawing social consensus than traditional social networks based on person-to-person relationships. We attempt to understand the BBS network from the perspective of a simple network model. In the model, we take into account the empirical fact that the BBS network contains groups of which size are inhomogeneous. In addition, the link density of each group is not uniform, however it decreases with increasing group size, which has been usually neglected when a model is constructed.

It would be interesting to implement the present work in the context of a previous study involving a psychosociological experiment on group discussions and the resulting consensus [1], in which, group discussions are distinguished into two types, "warm" and "cold." In the former type, people express their thoughts freely without any restriction, while in the latter, group discussions are restricted by some constraint either explicitly or implicitly, for example, the hierarchy in group members. The experimental study concludes that the consensus measured after group discussions can be different from that before the discussions depending on the type. In the former, the consensus after discussions shifts to an extreme opinions, while in the latter, it leads to a trade-off average group consensus. From the perspective of the experiment, we might state that the dialogues in the BBS are warm because no restriction is imposed on posting messages and little information on the personal background of the partner is provided. Thus, the dialogues in the BBS may lead to radicalized consensus, violent group behaviors, or imaginative and creative solutions to a given issue. Since students still in the process of developing a value system are vulnerable to negative influences, and have more opportunities to be influenced by their peers through online networks in this digital era than in the past, the proposed network pattern we report here will be useful in guiding them in the right direction. Moreover, the BBS network data will be helpful in understanding the manner in which diverse opinions are synchronized from the psychosociological perspective.

\section{ACKNOWLEDGMENTS}

This work was supported by KRF Grant No. R14-2002059-010000-0 of the ABRL program funded by the Korean government MOEHRD and the CNS from SNU (BK). 
[1] S. Moscovici and W. Doise, Dissensions et Consensus (Presses Universitaires de France, Paris, 1992); English edition as Conflict and Consensus (Sage, London, 1994).

[2] H. Ebel, L.-I. Mielsch, and S. Bornholdt, Phys. Rev. E 66, 035103(R) (2002).

[3] J. R. Tyler, D. M. Wilkenson, and B. A. Huberman, in Communities and Technologies, edited by M. Huysman, E. Wenger, and V. Wulf (Kluwer, Deventer, 2003).

[4] P. S. Dodds, R. Muhamad, and D. J. Watts, Science 301, 827 (2003).

[5] J.-P. Eckmann, E. Moses, and D. Sergi, Proc. Natl. Acad. Sci. U.S.A. 101, 14333 (2004).

[6] L. Adamic and E. Adar, Soc. Networks 27, 187 (2005).

[7] R. D. Smith, e-print cond-mat/0206378.

[8] R. Axelrod, J. Conflict Resolut. 41, 203 (1997).

[9] K. Klemm, V. M. Eguíluz, R. Toral, and M. San Miguel, Phys. Rev. E 67, 026120 (2003).

[10] P. S. Dodds, D. J. Watts, and C. F. Sabel, Proc. Natl. Acad. Sci. U.S.A. 100, 12516 (2003).

[11] F. Amblard and G. Deffuant, Physica A 343, 725 (2004).

[12] G. Deffuant, F. Amblard, and G. Weisbuch, Journal of Artificial Societies and Social Simulation (to be published).

[13] S. Milgram, Psychol. Today 2, 60 (1967).

[14] M. Granovetter, Am. J. Sociol. 78, 1360 (1973).

[15] S. Wasserman and K. Faust, Social Network Analysis
(Cambridge University Press, Cambridge, 1994).

[16] A. Barrat, M. Barthelemy, R. Pastor-Satorras, and A. Vespignani, Proc. Natl. Acad. Sci. U.S.A. 101, 3747 (2004).

[17] M. Girvan and M. E. J. Newman, Proc. Natl. Acad. Sci. U.S.A. 99, 7821 (2002).

[18] P. Holme, C. R. Edling, and F. Liljeros, Soc. Networks 26, 155 (2004).

[19] E. Adar, L. Zhang, L. A. Adamic, and R. M. Lukose, in Workshop on the Weblogging Ecosystem, 13th International World Wide Web Conference (ACM, New York, 2004).

[20] M. E. J. Newman, Proc. Natl. Acad. Sci. U.S.A. 98, 404 (2001).

[21] P. Holme, S. M. Park, B. J. Kim, and C. R. Edling, e-print cond-mat/0411634.

[22] S. Maslov and K. Sneppen, Science 296, 910 (2002).

[23] M. E. J. Newman, Phys. Rev. Lett. 89, 208701 (2002).

[24] L. C. Freeman, Sociometry 40, 35 (1977).

[25] M. E. J. Newman, Phys. Rev. E 64, 016132 (2001).

[26] K.-I. Goh, B. Kahng, and D. Kim, Phys. Rev. Lett. 87, 278701 (2001).

[27] Kou Zhougbao and Zhang Changshui, Phys. Rev. E 67, 036117 (2003).

[28] M. E. J. Newman, Phys. Rev. E 68, 026121 (2003).

[29] Y.-H. Eom et al. (unpublished).

[30] S. Valverde and R. V. Solé, e-print physics/0602005. 